\title{
Foundations of molecular similarity analysis
} Jürgen Bajorath

\author{
Address: Department of Life Science Informatics, B-IT, University of Bonn, Dahlmannstrasse 2, D-53113 Bonn, Germany \\ from 3rd German Conference on Chemoinformatics \\ Goslar, Germany. II-I3 November 2007 \\ Published: 26 March 2008 \\ Chemistry Central Journal 2008, 2(Suppl I):SI doi:I0.II86/I752-I53X-2-SI-SI
}

This abstract is available from: http://www.journal.chemistrycentral.com/content/2/SI/SI

(C) 2008 Bajorath

Rather different computational approaches are successfully employed to search for novel active compounds. Among those are various similarity-based methods that sometimes use only simple molecular representations. The complexity of computational tools and molecular representations does not correlate with their success in virtual screening and in recognizing increasingly diverse structures having similar activity. Why is this so? Why do many different virtual screening methodologies frequently display equally good or poor performance on given compound classes? We attempt to shed light on these open issues through systematic qualitative and quantitative characterization of underlying structureactivity relationships. Some surprises are ahead and the news is good, at least for virtual screeners and medicinal chemists. 